\title{
NUMERICAL STUDY OF THE PERFORMANCE LOSS OF A CENTRIFUGAL PUMP CARRYING EMULSION
}

\author{
Lila Achour $^{\text {a,b*, }}$, Mathieu Specklin ${ }^{\text {a }}$, Idir Belaidi ${ }^{\text {, }}$,Smaine Kouidri ${ }^{\text {a }}$ \\ a LIFSE, Arts et Métiers Institute of Technology, CNAM, HESAM University, Paris, France \\ ${ }^{\mathrm{b}}$ LEMI, FSI, University of M'hamed Bougara, Boumerdes, Algeria \\ * Correspondence author: Email: lila.achour@ensam.eu
}

Keywords: pump, emulsion, CFD, shear-thinning

\begin{abstract}
The performance and hydrodynamic behavior of centrifugal pumps when handling two-phase liquid-liquid flow and emulsion remain relatively unexplored, although they are of fundamental importance in optimizing the operating conditions of these pumps. Hence, this study aims at investigating the performance degradation of a centrifugal pump under emulsion flow by combined means of analytical and computational fluid dynamic (CFD) models. The analytical approach is based on internal energy loss equations while the CFD approach models the emulsion as a continuous and homogeneous single-phase fluid exhibiting shear thinning behavior. The results give a good insight into the performance degradation of such a system, especially at the best efficiency point (BEP).
\end{abstract}

\section{NOMENCLATURE}

$\begin{array}{ll}b & \text { empirical exponent } \\ c & \text { absolute velocity }(\mathrm{m} / \mathrm{s}) \\ C & \text { emulsion viscosity factor } \\ d & \text { diameter }(\mathrm{mm}) \\ e & \text { blade width }(\mathrm{mm}) \\ E & \text { empirical exponent } \\ F & \text { Area } \\ g & \text { gravitational acceleration }\left(\mathrm{m} / \mathrm{s}^{2}\right) \\ H & \text { head }(\mathrm{m}) \\ k & \text { rotation factor } \\ l & \text { length }(\mathrm{mm}) \\ L & \text { loss }(\mathrm{m}) \\ m & \text { empirical exponent } \\ n & \text { empirical exponent } \\ N & \text { stage number } \\ Q & \text { flow rate }\left(\mathrm{m}^{3} / \mathrm{s}\right)\end{array}$

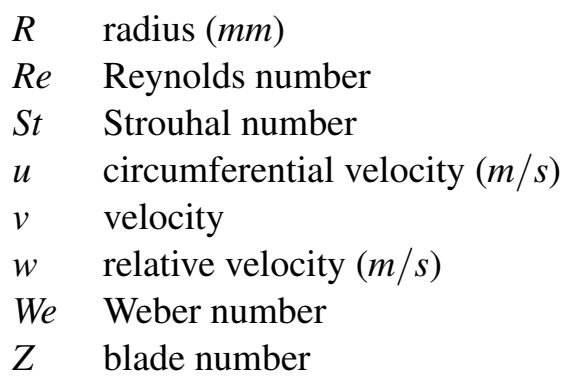

Greek symbols

$\beta \quad$ blade angle

$\varepsilon \quad$ wake coefficient

$\theta$ equivalent diffuser angle

$\lambda$ coefficient

$\mu \quad$ dynamic viscosity (Pa.s)

$\tilde{\mu} \quad$ ratio of oil viscosity to water viscosity

$\rho \quad$ density $\left(\mathrm{kg} / \mathrm{m}^{3}\right)$

$\varphi_{O I}$ oil volume fraction at inversion

$\varphi_{W I}$ water volume fraction at inversion

$\omega$ angular rotor velocity $(\mathrm{rd} / \mathrm{s})$

$\begin{array}{cl}\text { Subscripts } \\ 1 & \text { impeller blade leading edge } \\ 2 & \text { impeller blade trailing edge } \\ 3 & \text { volute cutwater } \\ 8 & \text { eight section of the volute } \\ 9 & \text { volute outlet } \\ \text { A } & \text { emulsion } \\ \text { d } & \text { design } \\ \text { c } & \text { casing or volute } \\ \text { dp } & \text { divergent part of the volute } \\ \text { e } & \text { blade or vane } \\ \text { E } & \text { effective } \\ \text { fr } & \text { friction } \\ \text { h } & \text { hydraulic }\end{array}$




$\begin{array}{ll}\text { im } & \text { impeller } \\ \mathrm{m} & \text { meridian } \\ \mathrm{M} & \text { mixture } \\ \text { mix } & \text { mixing } \\ \text { sep } & \text { separation } \\ \text { sh } & \text { shock } \\ \text { sp } & \text { spiral part of the volute }\end{array}$

\section{INTRODUCTION}

Centrifugal pumps find application in many engineering processes, chemical processes, wastewater treatment, power generation, and petroleum industries. They are generally designed to transport water or an incompressible fluid of low viscosity. Their selection is based on their performance, which is communicated by the pump manufacturer in the form of datasheets.

In certain engineering processes, the working fluids are a mixture of two immiscible liquids that form an emulsion. A typical example is the pumps used in the oil industry, where water is produced together with oil, causing the formation of crude oil-water emulsions. In such cases, the complex rheological behavior of liquidliquid two-phase flow and emulsion would significantly affect the flow pattern in the centrifugal pump and thus its performance. For instance, several studies have shown that the viscosity of the liquid-liquid dispersion and emulsion is higher than that of a single-phase fluid [1]. This viscosity depends essentially on the morphology of the emulsion and several parameters, such as the volume fraction of the coexisting phases, the size of the droplets of the dispersed phase, the shear stress, temperature, and the presence or not of a surfactant. The size of the droplets, as well as the presence of a surfactant, affect the stability of the emulsion, where it has been shown experimentally that the more stable the emulsion, the greater its viscosity [1], pointing out that high shear stress leads to droplet fragmentation and small droplet diameters, resulting in a stable emulsion [2]. As pointed out in [3], the increase in pump speed and flow rate, which increases the shear rate, promotes the fragmentation of particles due to high turbulence levels. On the other hand, the process of inter-conversion of phase configuration (phase inversion phenomenon) causes a dramatic rise in the emulsion viscosity, where it has been reported in the literature that the viscosity of the emulsion during the inversion process can be several times the viscosity of the single-phase oil constituting the emulsion. The pump performance initially deteriorates for rising dispersed phase volume fraction up to the inversion point, where a dramatic performance degradation is achieved at this zone. This complex rheological behavior that is accompanied by viscosity fluctuations affects the flow pattern in the centrifugal pump and thus its performance. In this regard, determining the performance of centrifugal pumps carrying emulsion becomes imperative.

Several computational investigations were conducted to study the centrifugal pump carrying emulsion by analyzing its overall performance, but no studies involved the rheological behavior of such fluids. This behavior is usually studied using rheological measurements to determine the viscosity as a function of the shear rate [4]. Many researchers have investigated the viscosity of emulsions at different water cut (WC) and found that in most cases, the emulsion exhibits a shear-thinning behavior beyond a critical water concentration [5]. In general, at higher water-oil ratios (WC $>30 \%$ ), the emulsion behaves as a pseudo-plastic fluid [6]. Hence, this study aims at investigating the performance degradation of a centrifugal pump handling emulsion flow by modeling the emulsion as a continuous and homogeneous single-phase fluid exhibiting a shear thinning behavior. This study also includes an analytical approach study which is based on internal energy loss equations coupled with an emulsion rheology model prediction, where a comparison between the results of the analytical and numerical model is reported.

\section{THEORETICAL ANALYSIS}

The analytical analysis is based on a rheological model of the emulsion in the pump proposed in the literature $[7,8]$, coupled with a loss model for pump performance prediction. The coupling considers the rheological model in order to estimate the effective viscosity of the emulsion as a function of operating conditions (variable flow rate in this study), which is then used in the friction coefficients and closure relationships in the pump performance model (Table 1).

\section{Emulsion rheology model}

The rheological model's first step is to evaluate the volume fraction of the dispersed phase at which phase inversion takes place (the dispersed phase becomes continuous), which is defined as follows [7, 8]: 


$$
\varphi_{O I}=\frac{\tilde{\mu}^{1 / E}}{1+\tilde{\mu}^{1 / E}}
$$

Or

$$
\varphi_{W I}=\frac{1}{1+\tilde{\mu}^{1 / E}}
$$

The following step is the application of the effective viscosity model of the emulsion, given by the relation:

$$
\mu_{A}=C\left(\mu_{E}-\mu_{M}\right)+\mu_{M}
$$

$\mathrm{C}$ is a factor that considers the effect of several parameters on the viscosity of the emulsion. It includes the effect of pump rotation speed, surface tension which affects the droplet size of the dispersed phase, and the oil viscosity making up the emulsion. The proposed form of $\mathrm{C}$ is as follow:

$$
C=\frac{(N W e R e)^{n}}{b S t^{m}}
$$

\section{Analytical model for pump head}

In this study, the analytical model of pump performance prediction is established by calculating theoretical head as well as losses. The loss mechanism within a centrifugal pump generally consists of friction, shock, separation, blade loading and wake mixing losses [9]. However, several correlations are available to estimate the different loss mechanisms [10]. Hence, an appropriate selection of these correlations is necessary to predict accurately the overall pump performance. The different loss mechanisms and models considered in this study are evaluated as described in Table 1.

\section{NUMERICAL MODEL}

The CFD simulations were performed with the opensource library OpenFOAM v1906 that uses a finitevolume method (FVM) to discretize the fluid equations. The computational domain consists of inlet and outlet suctions, impeller, and volute as shown in Fig. 1. The main geometrical dimensions of the centrifugal pump are summarized in Table 2.

Table 2

Main geometric parameters of the designed pump

\begin{tabular}{cccccccc}
\hline$R_{1}$ & $e_{1}$ & $\beta_{1}$ & $R_{2}$ & $e_{2}$ & $\beta_{2}$ & $Z$ & $R_{3}$ \\
\hline 75 & 85.9 & 70 & 204 & 42 & 63 & 5 & 218
\end{tabular}

\section{Mesh generation}

An unstructured mesh was generated due to the complex geometry of the centrifugal pump. Two types of mesh elements were considered: polyhedrons and prisms consisting of approximately 4 million cells in total. The hexahedral elements were employed in the inlet and outlet suctions, while the polyhedrons elements were employed in the impeller and volute. To capture the flow details near the flow domain boundaries, a structured mesh is used for the boundary layer of the rotating impeller. This leads to an average $y+<$ 5 and direct resolution of the viscous sublayer of the inner region. Fig. 2 gives a general view of the generated mesh.

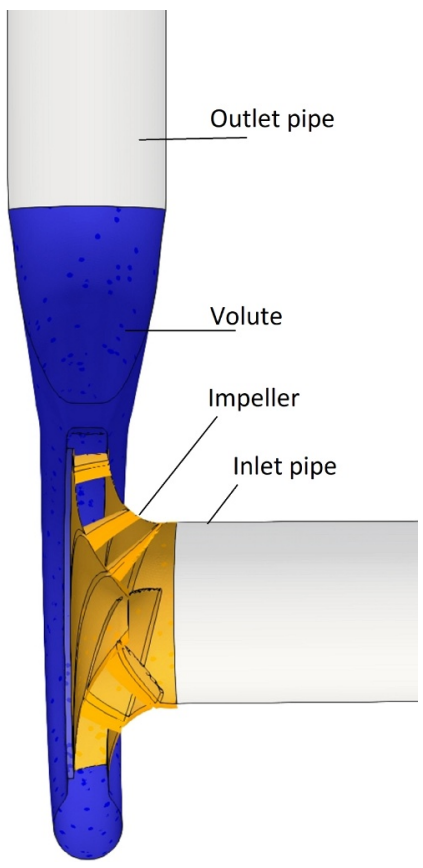

Figure 1

Pump 3-D model

\section{Emulsion rheology}

The emulsion considered in this study is a concentrated oil-in-water emulsion, composed of $40 \%$ oil volume fraction and $60 \% \mathrm{WC}$. Its rheological behavior was experimentally measured under a shear rate ranging from $1 s^{-1}$ to $3000 s^{-1}$ (data from reference [12]) that covers the range of the average shear rate of the studied pump. It was found that the studied emulsion has a low effective viscosity range with a clear tendency to shear thinning, fitting the Cross model. Thus, the numerical simulation of the emulsion flow within the pump assumes that the emulsion is a continuous and homogeneous single-phase fluid exhibiting a shear thinning 
Table 1

Loss mechanisms and corresponding correlation in a centrifugal pump

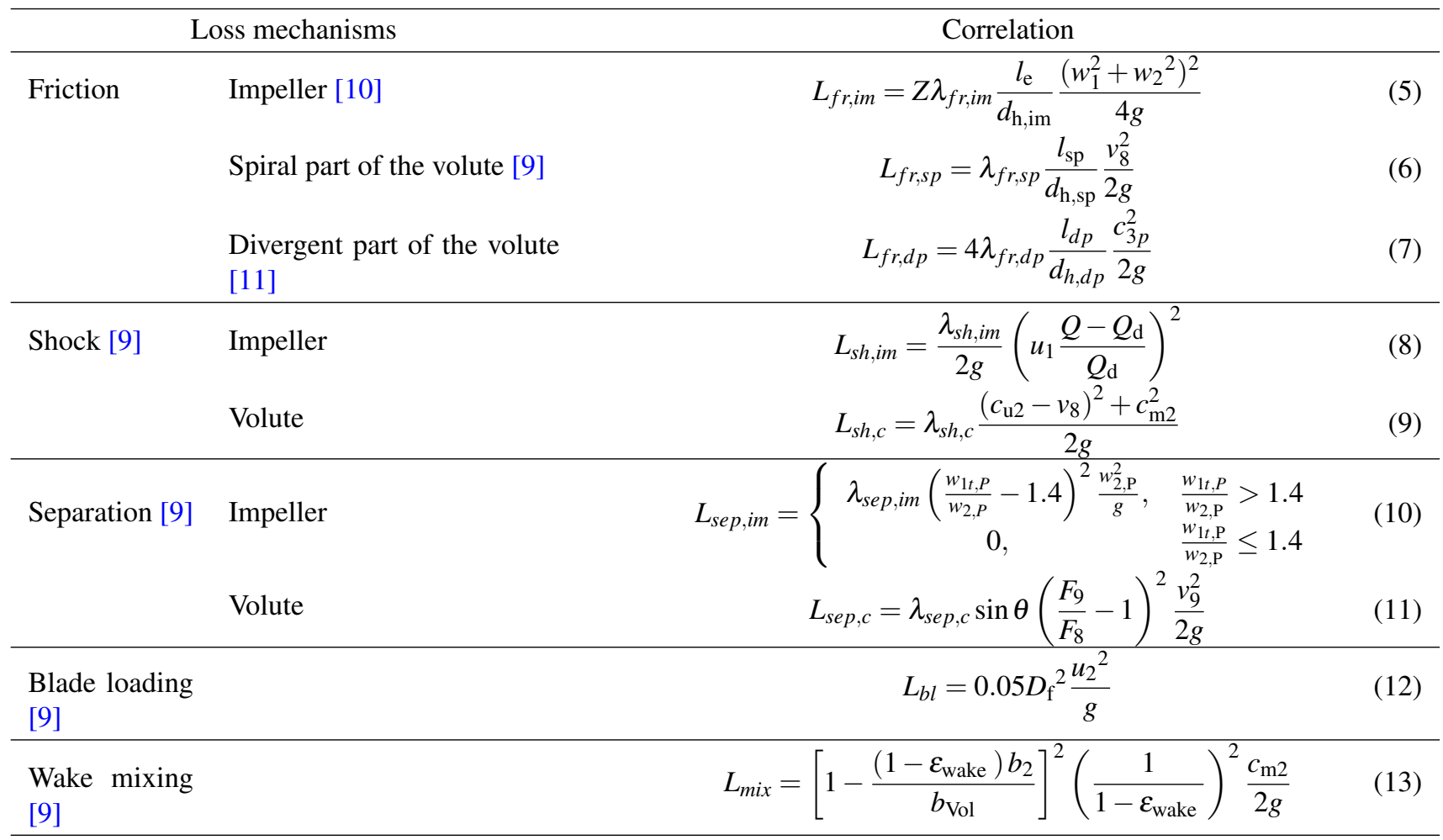

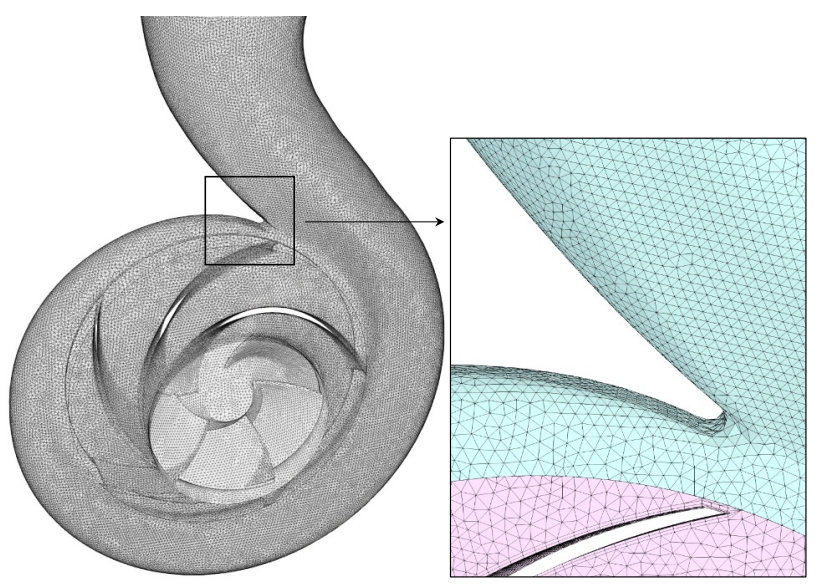

Figure 2

Fluid volume mesh

behavior. Its rheological behavior is described by the Cross model given by Eq. 14:

$$
\frac{\mu_{e f f}-\mu_{\infty}}{\mu_{0}-\mu_{\infty}}=\left[1+\left(K_{C} \dot{\gamma}\right)^{n_{C}}\right]^{-1}
$$

$\mathrm{k}$ and $\mathrm{n}$ are respectively the Cross time and Cross rate constant. $\mu_{0}$ and $\mu_{\text {inf }}$ are the viscosity for zero shear rate and a very high shear rate. Large k-values result in higher viscosity drops at low shear rates, resulting in stronger shear thinning. On the other hand, low n- values imply low viscosity drops at high shear rates, thus behaving more like a Newtonian fluid [13]. The studied emulsion and its properties are shown in Table 3 .

Table 3

Measured density and Cross fitting parameters of the studied emulsion from [12]

\begin{tabular}{ccccc}
\hline$\rho\left[\mathrm{kg} / \mathrm{m}^{3}\right]$ & $\mathrm{n}[-]$ & $\mathrm{k}\left[\mathrm{s}^{n}\right]$ & $\mu_{0}[$ Pa.s $]$ & $\mu_{\infty}[$ Pa.s $]$ \\
\hline 978.3 & 0.416 & 21.066 & 0.032 & 0.010 \\
\hline
\end{tabular}

\section{Simulation parameter}

In this study, the flow field within the centrifugal pump is assumed to be incompressible, single phase, viscous and turbulent. The mass and momentum equations (Eq. 15 and Eq. 16) are solved using the SIMPLE algorithm.

The Reynolds-averaged Navier-Stokes (RANS) equations are solved in ensemble-averaged form, including $\mathrm{k}-\varepsilon$ turbulence model for the effect of turbulence.

$$
\frac{\partial}{\partial t}(\rho)+\nabla \cdot(\rho \overline{\mathbf{u}})=0
$$

$$
\frac{\partial}{\partial t}(\rho \overline{\mathbf{u}})+\nabla \cdot(\rho \overline{\mathbf{u}} \otimes \overline{\mathbf{u}})=\mathbf{g}+\nabla \cdot \overline{(\tau)}-\nabla \cdot(\rho \mathbf{R})
$$


$\bar{\tau}$ is the averaged stress tensor and $\mathbf{R}$ is the Reynolds stress tensor. The Reynolds stress terms in RANS equations are modeled by the two transport-equation lineareddy-viscosity turbulence closure model $\mathrm{k}-\varepsilon$. The solved turbulent kinetic energy equation $\mathrm{k}$ and dissipation rate equation $\varepsilon$ are given by Eq. 17 and Eq. 18 . The motion of the rotational domain is modeled using the multiple reference frame (MRF) technique, in which the impeller is in the rotating frame, and the volute and the rest of the pump geometry in the stationary frame. The rotation speed of the impeller is 900 rpm. The information between the different regions is transferred by an arbitrary mesh interface (AMI). A first-order discretization scheme was used for the turbulent terms and a second-order discretization scheme for the convective and diffusive terms. The inlet and outlet boundary conditions were set to volumetric flowrate and static pressure of $0 \mathrm{~Pa}$, respectively and a no-slip velocity condition is imposed on all pump walls. By changing the volumetric flow rate, the performance curves of the centrifugal pump were acquired.

$$
\frac{D}{D t}(\rho k)=\nabla \cdot\left(\rho D_{k} \nabla k\right)+P-\rho \varepsilon
$$

$$
\begin{gathered}
\frac{D}{D t}(\rho \varepsilon)=\nabla \cdot\left(\rho D_{\varepsilon} \nabla \varepsilon\right)+\frac{C_{1} \varepsilon}{k}\left(P+C_{3} \frac{2}{3} k \nabla \cdot \mathbf{u}\right)-C_{2} \rho \frac{\varepsilon^{2}}{k} \\
v_{t}=C_{\mu} \frac{k^{2}}{\varepsilon}
\end{gathered}
$$

\section{RESULTS AND DISCUSSION}

\section{Analytical and numerical model validation}

In order to estimate the accuracy of both analytical and CFD models, a standard case with water only was first considered. Fig. 3 shows the comparison of the pump head predicted by the two models and the experimental data as a function of flow rate. Excellent agreement with the experimental data can be observed for the head predicted by the analytical model with a deviation of less than $5 \%$ at low and high flowrates. Although the trend is well reproduced, the numerical model underestimates the head values compared to the experimental data. Nearly all data points are within $10 \%$ discrepancy. The differences can be related to the numerical modeling errors and the inability of reproducing the pump design in detail. The results indicate that the hydraulic loss equations used for pump head prediction are correctly evaluated and that CFD can predict pump performance with a satisfying accuracy.

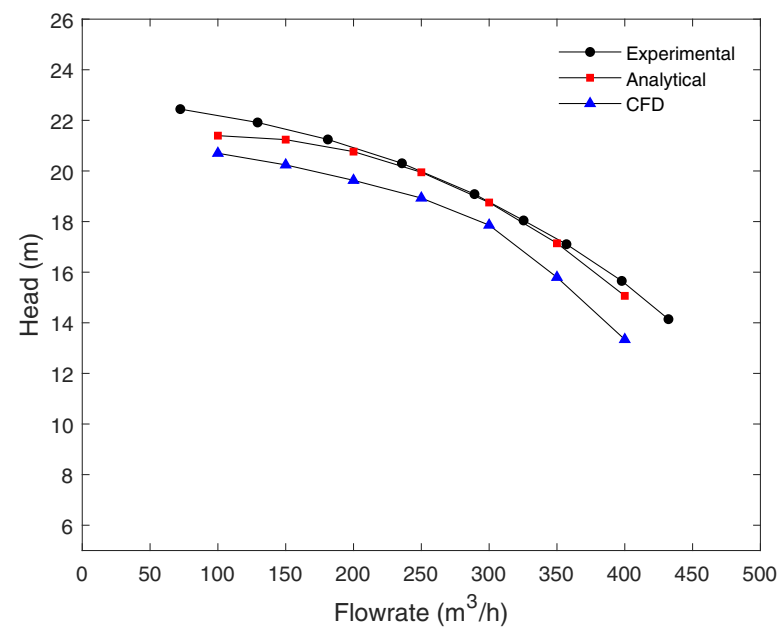

Figure 3

Comparison of pump head predicted by the two models under water flow at $900 \mathrm{rpm}$ with experimental data.

\section{Pump flow field distribution}

Fig.4 (a-c) shows the emulsion relative velocity profiles and streamlines in the impeller at $900 \mathrm{rpm}$ for different flow rates. At the nominal point $\left(300 \mathrm{~m}^{3} / \mathrm{h}\right)$, the velocity is uniformly distributed in the different channels of the impeller, while a considerable variation is observed under off-design operation conditions. Near the trailing edges of the impeller blades, recirculation zones can be noticed. Large vortices increase recirculation losses, thus reducing pump performance. As pointed out in [14], two factors affect the recirculation loss, including the fluid viscosity and the off-design operation conditions.

\section{Emulsion effective viscosity variation and shear stress profiles}

Fig. 5 shows the emulsion viscosity predicted by the rheological model presented in the literature using Zhu [8] and Banjar [7] coefficients as a function of water cut at $\mathrm{BEP}\left(900 \mathrm{rpm}\right.$ and $\left.\mathrm{Q}=300 \mathrm{~m}^{3} / \mathrm{h}\right)$, compared to the experimental data from [12]. A clear peak in the emulsion viscosity is observed as the water-volume fraction increases, indicating the reversal of the continuous-oil emulsion (water-in-oil) to a continuous-water emulsion (oil-in-water), resulting in a significant increase in its effective viscosity. From the figure, the phase inversion point predicted by both models is lower than the experimental one (20\% WC vs. $30 \% \mathrm{WC}$ in experimental data), highlighting also that Banjar's model gives a better approximation of the viscosity value at this point. Before the phase inversion, the experimental data are 

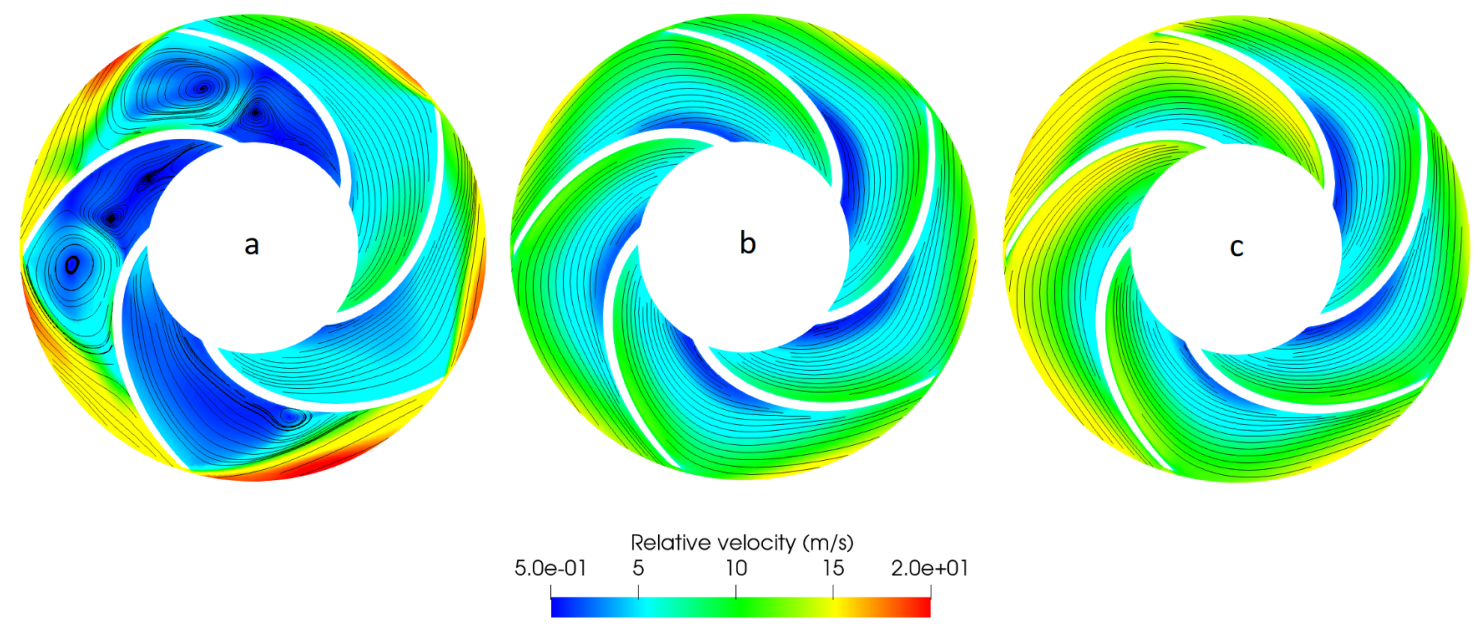

Figure 4

Relative velocity profile and streamlines for emulsion operating at: (a) 0.5 BEP, (b) BEP, and (c) 1.2 BEP.

well predicted by Banjar's model with a deviation of $8 \%$. Conversely, after the inversion point, the experimental data are well predicted by Zhu's model with a discrepancy under $14 \%$. The difference between the predicted inversion point and the experimental one is expected since the rheological model is semi-empirical. The proposed equation for inversion point determination (Eq. 2) depends on the viscosities of the two coexisting phases, linked by an exponent $\mathrm{E}$ determined experimentally. The operating conditions of this study and the properties of the oil constituting the emulsion (viscosity and surface tension) differ from those of the two authors, therefore the exponent E can not be applied in this case. Besides, exponent $\mathrm{E}$ is determined for fixed operating conditions and is applied over the range of flow rates and speeds relative to the studied pump. It has been reported that phase inversion depends on the properties of the emulsion (continuous phase oil viscosity and emulsion stability) as well as the shear rate applied to the emulsion (rotational speed and flow rate). Phase inversion occurs at a higher volume fraction of the dispersed phase as the rotational speed increases and the viscosity of the oil continuous phase decreases [15]. The proposed rheological model checks the effect of viscosity on phase inversion but does not reflect the operating conditions or emulsion stability effect. Consequently, the viscosity model (Eq. 2 and Eq. 4) should be fitted to the experimental data used in this paper to give a better prediction of the inversion point as well as the overall emulsion viscosity.

In Fig. 5, the rheological model with adjusted exponents is plotted against the experimental data as a function of WC. The adjustment consisted of increasing the

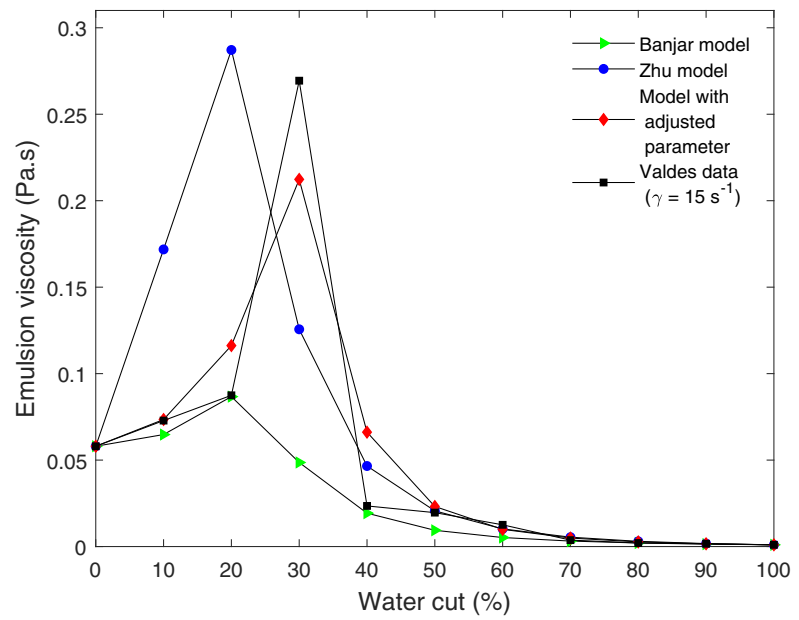

Figure 5

Comparison of the emulsion effective viscosity predicted by the rheological model using Zhu [8] and Banjar [7] coefficients with the experimental data of [12] and the adjusted rheological model

exponent E (Eq. 2) (compared to the other models) to account for the difference in operating conditions (lower specific speed and larger pump size compared to the reference papers). The fitted model gives an accurate prediction for the inversion point, however the viscosity is underestimated with a difference of $20 \%$. Outside the phase inversion zone (a 20\% margin before and after the inversion point), the model gives an accurate prediction of the emulsion viscosity with a difference of less than $9 \%$.

As mentioned previously, the emulsion considered in this study is water-continuous with $60 \% \mathrm{WC}$. The predicted viscosity for these volume fractions using the analytical model is $1.1310^{-3}$ Pa.s against $1.2510^{-3} \mathrm{~Pa} . \mathrm{s}$ 

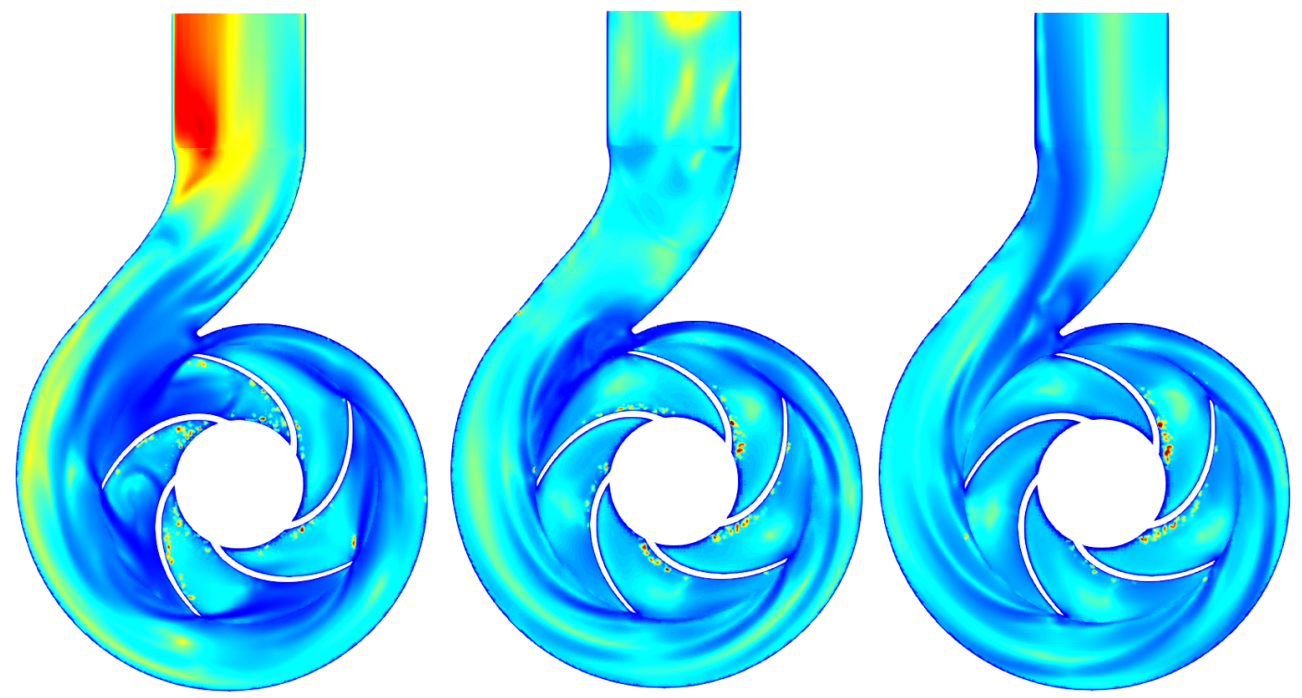

Effective viscosity $\left(\mathrm{m}^{2} / \mathrm{s}\right)$

1.0e-05 $\quad 1.1 e-5 \quad 1.2 e-5 \quad 1.3 e-05$

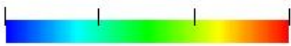

Figure 6

Effective viscosity (a-c) for emulsion operating at: (a-d) 0.5 BEP, (b-e) BEP, and (c-f) 1.2 BEP.

in experimental data at BEP. On average, the viscosities vary from $0.7210^{-3}$ to $1.3010^{-3} \mathrm{~Pa}$.s as the flow rate varies from 100 to $400 \mathrm{~m}^{3} / \mathrm{h}$. The analytical model of viscosity gives an approximate estimation of the emulsion rheology but does not describe the fluid behavior within the pump. Therefore, the rheological behavior and shear stress profiles are analyzed using CFD. Fig.6 and 7 (a-c) show the emulsion viscosity on the pump at different flowrates. As the flow rate increases, the emulsion viscosity decreases, which is clearly observed at the outlet section of the pump, slightly on the blade surfaces and at the impeller inlet. This is due to the higher shear rate applied to the fluid at higher operating flow rates, causing a decrease in emulsion viscosity; which is consistent with the fluid shear-thinning behavior. This shear-thinning behavior is further emphasized in Fig. 7 (d-f), which shows the shear stress profile in the impeller at different flow rates. The high shear stress regions in Fig. 7 (d-f) (impeller leading edge and blade surface) correspond to the low viscosity region in Fig. 7 (a-c). Besides, the effective viscosities observed at the outlet section and the volute are significantly higher than the ones observed at the rotational regions. In sum, larger viscosities are observed far from the impeller caused by the lower shears exerted on the fluid at that region.
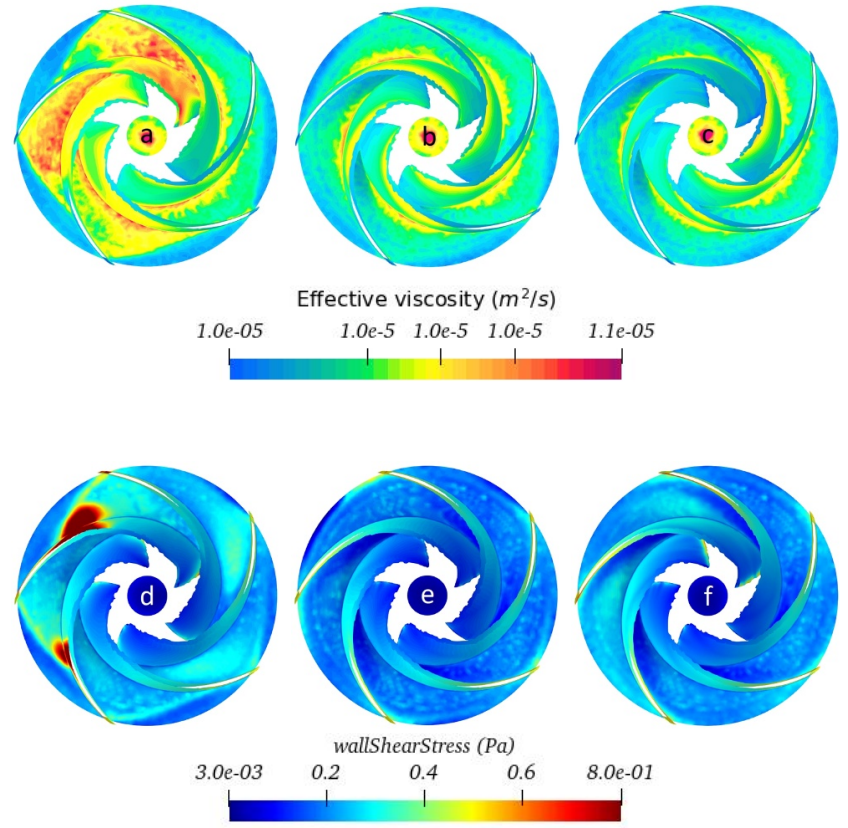

Figure 7

Effective viscosity profile $(a, b, c)$ and Shear Stress profiles (d, e, f) on the impeller operating at: (a-d) 0.5 BEP, (b-e) $\mathrm{BEP},(\mathrm{c}-\mathrm{f}) 1.2 \mathrm{BEP}$

The maximum viscosity of the emulsion at the impeller is about $1.110^{-3}$ Pa.s, while this value is about 1.3 $10^{-3}$ Pa.s at the volute. These results highlight that the viscosity upper limit value from Cross correlation is not reached even at very low flow rates. Furthermore, in this study, only a slight viscosity variation occurs in 
the pump impeller, noting a variation ranging from 1.02 $10^{-3}$ to $1.310^{-3}$ Pa.s. This suggests that the shear rate in the rotating zone is dominated by rotational speed and is less sensitive to flow variations. In contrast, in the other regions of the pump, the shear rate is highly sensitive to flow variations.

\section{Centrifugal pump performance}

Fig. 8 shows the pump head calculated analytically and numerically when operating with water and emulsion. Since the emulsion viscosity is higher than that of water, the pump head decreases, which is caused by an increase in hydraulic losses, mainly friction one as outlined in previous studies [13]. Both the analytical and numerical models show that the degradation of the pump head caused by the rheological behavior of the emulsion increases with increasing flow rate. Pointing out that the numerical model gives a significant degradation when comparing with the degradation given by the analytical model. This is due to the fact that the viscosity of the fluid is considered only in the friction loss equations in the analytical model. Moreover, the analytical model is semi-empirical and finds its limits when the fluid is very viscous.

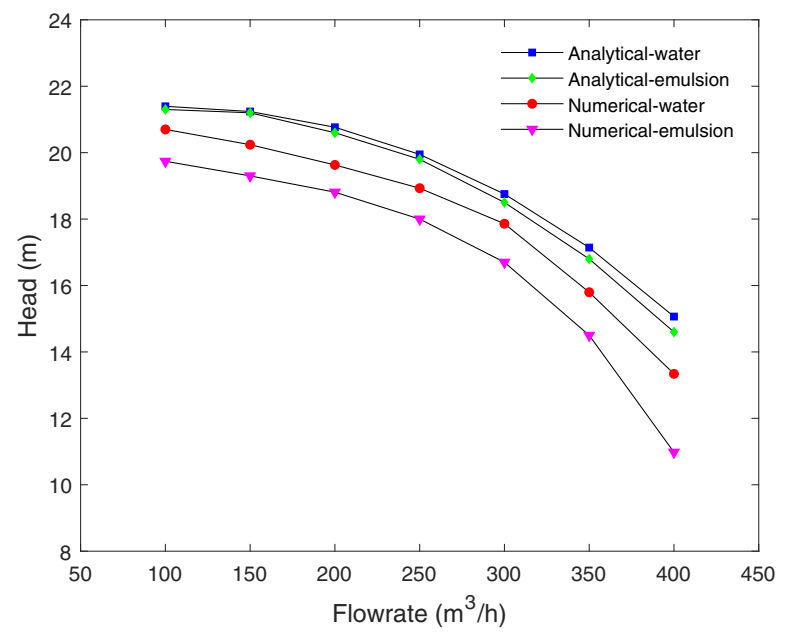

Figure 8

Analytical and numerical pump head results for water and emulsion at $900 \mathrm{rpm}$

In this study, no further conclusion can be made regarding the accuracy of the models or which model gives better performance prediction given the lack of experimental results. However, a comparison between the CFD results and experimental data of the reference article is presented in Fig. 9. It shows the normalized head versus normalized flow for CFD results and Valdes ex- perimental data based on a multistage pump having a specific speed twenty times the specific speed of the studied pump. As it can be noticed, the trend of the graph is similar for both results. Another interesting observation is the head deteriorating rate. The head curve for emulsion flow depicted in Fig. 8 shows a more pronounced drop at higher flowrates. This observation is in accordance with the experimental results of Valdes [12], where a $4 \%$ performance degradation was noted before BEP, increasing up to $14 \%$ at this point. In this study, the degradation rate is $9 \%$ before BEP and increases up to $17 \%$ after this point. First, this difference of deterioration can be attributed to the difference in pump geometry and operating conditions that affect the hydraulic losses in terms of quantity. Second, despite the shear-thinning behavior of the emulsion, its high effective viscosity leads to degradation of pump performance, primarily through increased frictional losses at high flow rates.

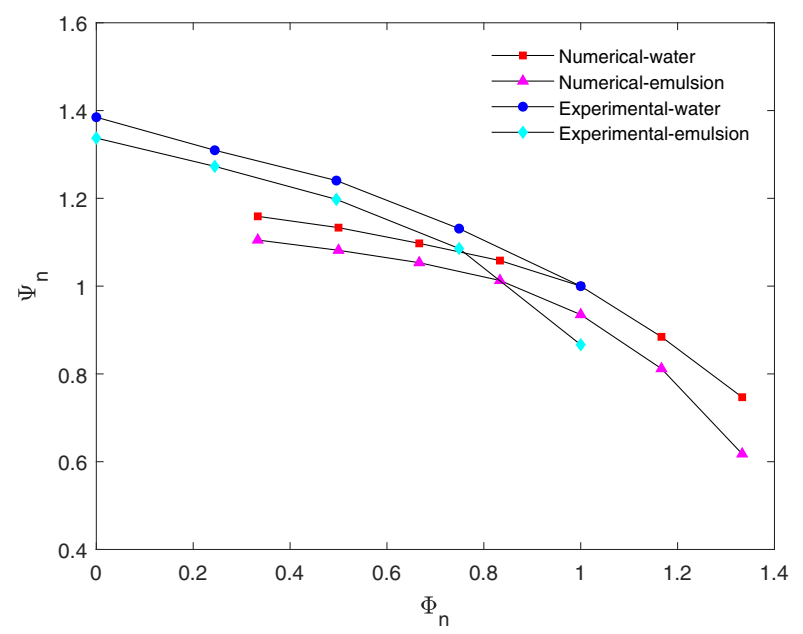

Figure 9

Curves of normalized head coefficient as a function of the normalized flow coefficient for CFD results and Valdes experimental data

\section{CONCLUSIONS}

In this study, an analytical and CFD analysis were performed to analyze the performance of a centrifugal pump when handling a concentrated oil in water emulsion. The analytical model is a coupling between a model for predicting the emulsion viscosity and a model for predicting the pump performance based on loss mechanism. While the CFD model assumes that the emulsion is a homogeneous single-phase fluid exhibiting shear-thinning behavior. The conclusions of this study are summarized below: 
1 The rheological model proposed in the literature gives a good approximation of the emulsion viscosity outside the inversion zone after fitting the exponents to the experimental data.

2 Pump performance predicted by the numerical simulations and the analytical model shows a good agreement near BEP while higher deviations were noticed at off-design conditions.

3 The pump head curves obtained from CFD simulations showed a steeper drop at higher flow rates when handling an oil-in-water emulsion, which is consistent with the literature results.

4 Modeling the emulsion as a homogeneous singlephase fluid with appropriate rheology can be a convenient method to estimate the overall pump performance without considering the multiphase topology of the fluid.

\section{REFERENCES}

[1] MF Khalil, SZ Kassab, AS Ismail, and IS Elazab. Centrifugal pump performance under stable and unstable oil-water emulsions flow. Twelfth International Water Technology Conference, (January):687-702, 2008.

[2] Philipp Schmitt, Mark W. Hlawitschka, and Hans Jörg Bart. Centrifugal Pumps as Extractors. Chemie-Ingenieur-Technik, 92(5):589-594, 2020.

[3] Rodolfo Marcilli Perissinotto, William Monte Verde, Carlos Eduardo Perles, Jorge Luiz Biazussi, Marcelo Souza de Castro, and Antonio Carlos Bannwart. Experimental analysis on the behavior of water drops dispersed in oil within a centrifugal pump impeller. Experimental Thermal and Fluid Science, 112(August 2019):109969, 2020.

[4] Diego Pradilla, Watson Vargas, and Oscar Alvarez. The application of a multi-scale approach to the manufacture of concentrated and highly concentrated emulsions. Chemical Engineering Research and Design, (February):1-11, 2014.

[5] Yazhou Zhou. A comprehensive review of emulsion and its field application for enhanced oil recovery. (January):1046-1058, 2019.
[6] Fatemeh Goodarzi and Sohrab Zendehboudi. A Comprehensive Review on Emulsions and Emulsion Stability in Chemical and Energy Industries. Canadian Journal of Chemical Engineering, 97(1):281-309, 2019.

[7] Hattan M. Banjar. Experiments, cfd simulation and modeling of oil viscosity and emulsion effects on esp performance. The University of Tulsa, page 121, 2018.

[8] Jianjun Zhu, Haiwen Zhu, Guangqiang Cao, Hattan Banjar, Jianlin Peng, Qingqi Zhao, and Hong Quan Zhang. A new mechanistic model for oil-water emulsion rheology and boosting pressure prediction in electrical submersible pumps ESP. Proceedings - SPE Annual Technical Conference and Exhibition, 2019-Septe(January), 2019.

[9] Ming Liu, Lei Tan, and Shuliang Cao. Theoretical model of energy performance prediction and bep determination for centrifugal pump as turbine. Energy, 172:712-732, 2019.

[10] Tatiane Silva Vieira, José Ricardo Siqueira, André Duarte Bueno, Rigoberto E.M. Morales, and Valdir Estevam. Analytical study of pressure losses and fluid viscosity effects on pump performance during monophase flow inside an ESP stage. Journal of Petroleum Science and Engineering, 127:245-258, 2015.

[11] Abdellah Kara Omar, Abdelkrim Khaldi, and Abdelkrim Ladouani. Prediction of centrifugal pump performance using energy loss analysis. Australian Journal of Mechanical Engineering, 15(3):210-221, 2017.

[12] Juan Pablo Valdés, Miguel Asuaje, and Nicolás Ratkovich. Study of an ESP's performance handling liquid-liquid flow and unstable O-W emulsions Part I: Experimental. Chemical Engineering Science, 223, 2020.

[13] Juan Pablo Valdés, Deisy Becerra, Daniel Rozo, Alexandra Cediel, Felipe Torres, Miguel Asuaje, and Nicolás Ratkovich. Comparative analysis of an electrical submersible pump's performance handling viscous Newtonian and non-Newtonian fluids through experimental and CFD approaches. 
Journal of Petroleum Science and Engineering, 187(November 2019), 2020.

[14] Jianjun Zhu, Hattan Banjar, Zhenyan Xia, and Hong Quan Zhang. CFD simulation and experimental study of oil viscosity effect on multi-stage electrical submersible pump (ESP) performance. Journal of Petroleum Science and Engineering, 146:735-745, 2016.

[15] Natan Augusto Vieira Bulgarelli, Jorge Luiz Biazussi, Marcelo Souza De Castro, William Monte Verde, and Antonio Carlos Bannwart. Experimental study of phase inversion phenomena in electrical submersible pumps under oil water flow. Proceedings of the International Conference on Offshore Mechanics and Arctic Engineering OMAE, 8:1-8, 2017. 